\title{
Cyber-Physical Production Management
}

\author{
Guenther Schuh, Till Potente, Christina Thomas, and Annika Hauptvogel \\ Laboratory for Machine Tools and Production Engineering (WZL) \\ of RWTH Aachen University, Aachen, Germany \\ \{g.schuh, t.potente, c.thomas, a.hauptvogel\}@wzl.rwth-aachen. de
}

\begin{abstract}
Today a high adherence to delivery dates is the main logistic target for manufacturing companies. To control the increasing complexity of production planning and control manufacturing companies use IT systems. However, the applied IT systems often do not provide a reliable forecast of delivery dates and thus affect the required adherence to delivery dates. The paper describes a cyber-physical approach to optimize the production planning and control towards a reliable detailed planning. Therefore, it is necessary to determine the deviations between the production schedule of the IT system and the production processes in reality. With the knowledge of the revealed deviations and their causes the process of production planning can be adjusted towards a more dependable high resolution production planning and control.
\end{abstract}

Keywords: production planning, production control, simulation model.

\section{$1 \quad$ Introduction}

The central challenge for manufacturing companies is to handle the increasing dynamics of markets and individual customer demands [1-2]. It becomes apparent that the production planning and control (PPC) is the key element in meeting these requirements [3-5]. Due to these trends the complexity in planning manufacturing processes is increasing constantly. However, the behavior of complex systems cannot be predicted in detail [6]. Therefore, the use of IT systems (e.g. Advanced Planning and Scheduling systems, APS systems) has become necessary for manufacturing companies to support the PPC [7]. The use of such IT systems often does not predict the future situation of the production reliably. Instead, the use of these IT systems quickly leads to complex structures in PPC. Hence, the transparency and flexibility of order processing is no longer available for the company [8]. The results of several analyses of the Laboratory for Machine Tools and Production Engineering (WZL), especially in companies with individual and small series production, demonstrated that the implemented APS system has deficiencies regarding the forecasting reliability of the planning processes. The start dates of each production step of any product are corrected continuously over the entire planning period until the completion of the product. The dates are usually adjusted upwards, whereby the delivery date is delayed. Thus the adherence to delivery dates is decreasing. Due to a lacking transparency of IT systems, causes for deviations are not traceable for the practical planner. 
One reason for such deviations is that changes in the real production system are not adapted adequately in the IT system. Adjustments require expertise and a lot of time. To ensure an effective planning in the future, deviations between the planning of the IT system and the real production must be uncovered. This is necessary in order to ensure a reliable planning as well as the confidence of the practical planner in the planning of the IT systems.

\section{State of the Art}

The manufacturing processes are not only complex but also chaotic. The behavior of complex systems cannot be predicted or only for a short period [9]. The value of scientific knowledge is the ability to make predictions for the future. These predictions become problematic in case of complex systems with chaotic processes. Similar causes can have completely different effects. Even in case of nearly identical facts, the effects are different [10].

\subsection{IT Systems to Support Production Planning and Control}

APS systems were established at the end of the 90 s to increase the benefit of IT systems in manufacturing processes by improving the planning systematically. APS systems use exact mathematical optimization techniques and heuristics to predict the future production schedule. The specific type of planning model and the solution algorithm used to calculate the production schedule in general are not transparent for the practical planner [11]. With the models implemented in the IT systems today the current situation of the production is not depicted in the systems, because the model is defined, when the system was implemented the first time. The initial version needs to be adapted frequently because of new products or variants, new machines or process optimizations. In many companies these adaptions are not executed, because they demand manual expense [12]. The planning carried out by the system cannot be correct under these conditions and deviates from the reality in the production. In addition to the initial predetermined master data the control strategies, for example for sequencing, are also permanently defined. An optimal configuration of the control strategy is not provided by current APS systems. Simultaneously, a solution of the possible combinations of control strategies regarding the dependencies of individual jobs and machines is too complex for humans.

\subsection{Cyber-Physical Principles in Production Planning and Control}

To handle the challenges described in the beginning a promising approach is described by cyber-physical systems (CPS). The term CPS refers to a new generation of systems with integrated computational and physical capabilities that can interact with humans through frictionless user interfaces [13]. Thus, humans making decisions are supported optimally. Moreover, CPS meet the necessary requirements for the realization of a 
reliable planning. They are characterized mainly by an increased computing power that is needed to perform as many different simulations simultaneously [14]. Using appropriate sensor technology, CPS are able to directly receive physical data as well as using all the available data by connecting through digital networks [15]. To determine the initial conditions of the model largely accurate, it is important that relevant factors (e.g. production data, machine data, etc.) are recorded as precise as possible in production. By the use of CPS and the corresponding intelligent sensor technologies access to more data is enabled and therefore a more accurate determination of the initial conditions is possible.

\section{Requirements}

Nowadays companies rely on detailed planning systems to manage the complexity of the production and to support the PPC. Requirements for such IT systems include a reliable statement on the completion date of all orders and thus the reliable attainment of the customer agreed date. Since date adherence is the leading logistic target in manufacturing companies [16], it is essential to meet the customer agreed date. If a reliable planning of the detailed planning system is ensured, the production controller will be able to shift his focus from the prioritization of delayed orders in order to realize the dates agreed by the customer. Instead, he can focus on optimizing the PPC.

Therefore three key issues have to be regarded:

- How can deviations between the production schedule, determined by the IT system, and the production processes in reality be identified?

- How can a simulation model be optimized depending on the identified deviations to reflect reality?

- How can a reliable prediction of the future situation in the production be made using the optimized simulation model?

These requirements for future detailed planning systems must be met in the near future so that manufacturing companies can succeed on a global market. In the following these requirements will be regarded by describing the approach of cyberphysical production management.

\section{Cyber-Physical Production Management}

\subsection{Step 1: Identification of Deviations}

The approach of cyber-physical production management is based on statistical modeling using simulation. The idea is to implement a judgment system (second-order cybernetics), which monitors the deployed IT systems (ERP system, APS system etc.), as it is displayed in figure 1. Therefore, it is necessary for the judgment system to get information from the ERP system about current orders and work schedules. In addition, information of the detailed scheduling system (APS system or MES) about its 
current planning is essential, too. The first challenge is the storage of the planning, because today the current planning is overwritten after every planning run. This is solved by implementing a so called data-collector, which stores the data history of every day plans in a database.

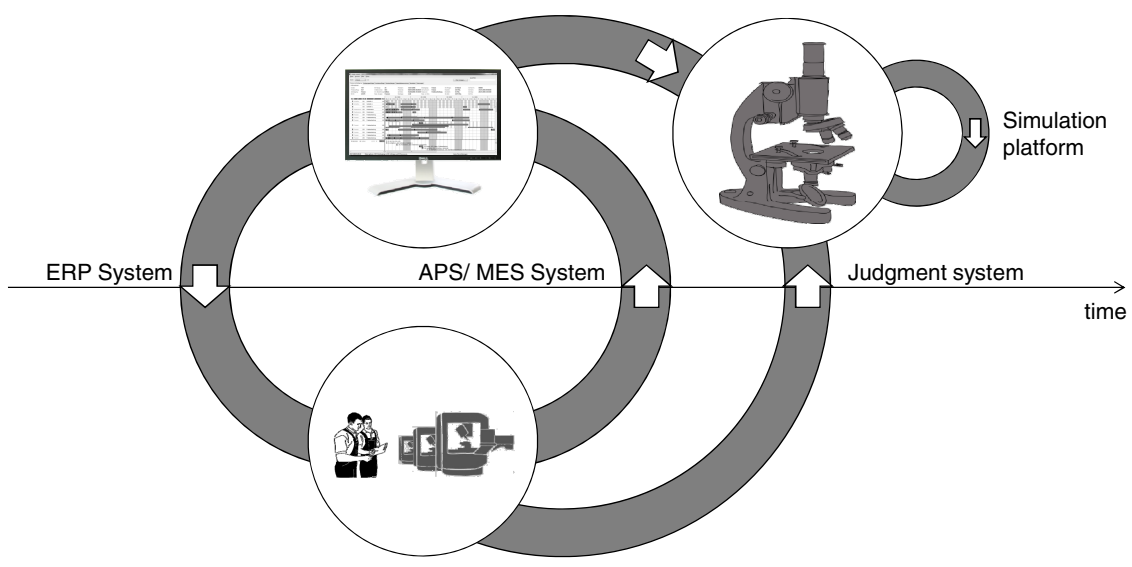

Fig. 1. Approach of cyber-physical production control [17]

The third important information is the feedback data of the production (production data logging, machine data logging etc.). Due to intelligent sensor technologies of CPS the feedback data can be specified. With the stored plans on the one hand and the knowledge about the resulted situation in the production on the other hand the judgment system has every information it needs to compare the systems planning and the reality on the shop floor. By comparing the plan of the detailed scheduling system and the real situation deviations from the plan can be determined. Therefore, every order position is regarded in the plan and the feedback data for an interval, which is predetermined by the frequency of the data-collector. The practical planner gets the information on how good the detailed scheduling system matches with the reality on the shop floor and how good the model is that he is using for production control, which is implemented in the system.

\subsection{Step 2: Best Fitting Simulation Model}

As it is shown in figure 1, the judgment system is supported by a simulation platform. After the quality of the planning of the detailed scheduling system is judged in the first step, the question to be answered is how to get a model displaying the production system more adequately. Therefore, a simulation model is build with defined parameters for:

- Order release,

- sequencing and

- capacity control. 
The implemented model is parameterized with the feedback data of the production (High-resolution Data Collection) for a significant period. After each simulation run, the result is compared to the feedback data as it has been done before with the planning of the detailed scheduling system. Thus, the quality of the simulation model is determined. Depending on the result the parameters of the model are adapted (Optimization) and the simulation is run again (Simulation). This control loop is displayed by the inner control loop in figure 2 . The outer control loop describes the aim of every company to constantly optimize their production system to achieve their targets superiorly. The more simulation runs are made with improved parameters and compared to the real situation on the shop floor the better the simulation model gets. The increasing computational power enabled by cyber-physical systems facilitates more concurrent simulations in less time.

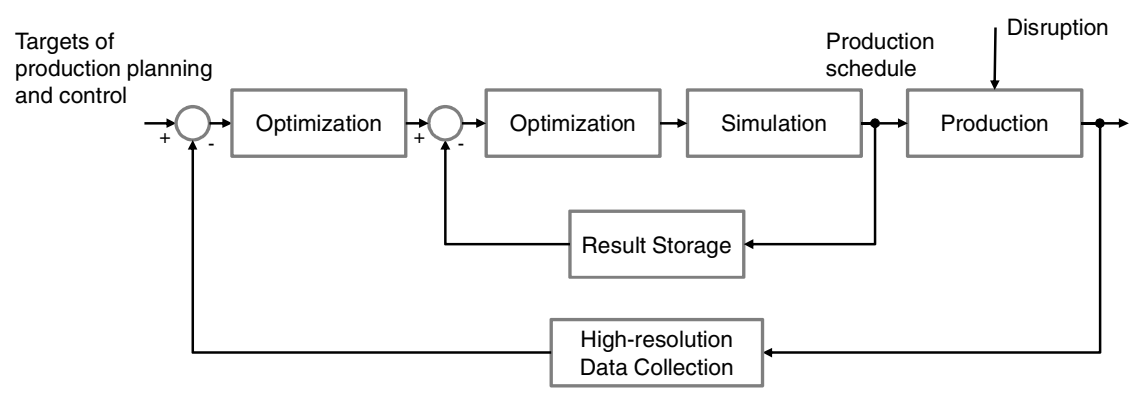

Fig. 2. Architecture of cyber-physical production control

\subsection{Step 3: Planning by Simulation}

With the resulting best fitting model and data of future orders provided by the ERP system a new planning can be enabled, which has a more exact prediction accuracy. This was analyzed by data of a company with individual and small series production. As described in 4.1 the deviations between the detailed scheduling system and the reality on the shop floor are determined. The planning reliability of the detailed scheduling system was by only $25 \%$ after 3 days in comparison to the real situation on the shop floor, which is displayed in the left part of figure 3. Afterwards, we implemented a simulation model with following parameters:

- Order release: Date planned by the applied IT system

- Sequencing: FiFo (First-in-First-out)

- Capacity control: According to shift schedule

The simulation model was run with these parameters and compared to the real situation on the shop floor during the same interval. The result, shown in figure 3 on the right side, demonstrates that the planning done by the implemented simulation model shows better results in comparison to the real production even with these simple parameters. 

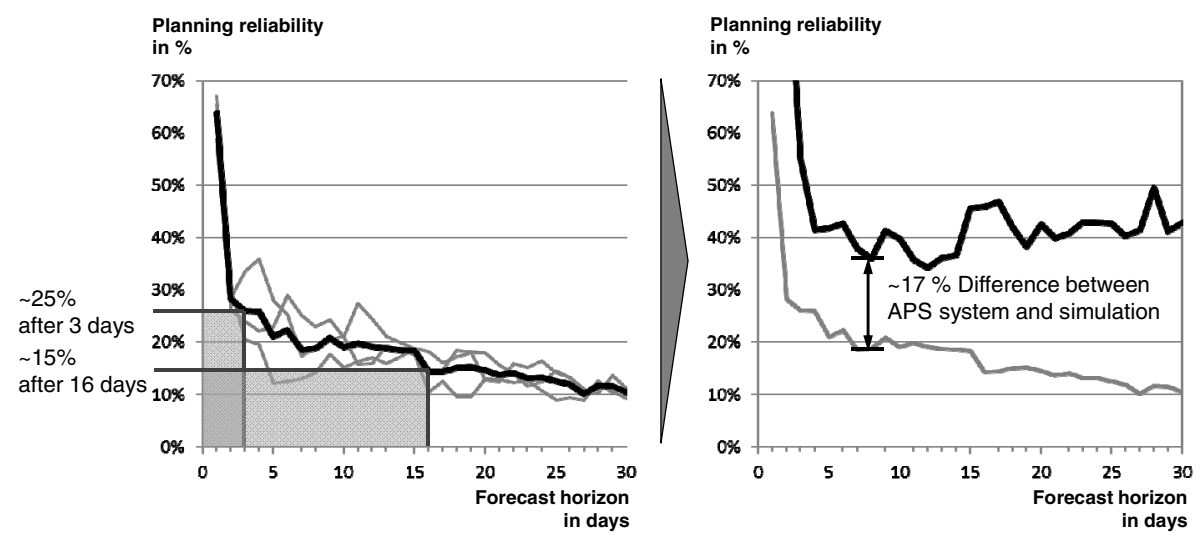

Fig. 3. Planning reliability of APS system and simulation

\section{$5 \quad$ Outlook}

\subsection{Measuring of the Reliability}

Figure 3 illustrates that a more reliable planning of production processes is possible, if the current situation on the shop floor is implemented in the model, which is applied to run the planning. The quality of the planning done by simulation runs depends on the quality of the particular simulation model. The simulation model is described by the feedback data. Thus, the information describing the situation on the shop floor affects the quality of the simulation model and therefore the planning.

The practical planner has to make his decision based on the planning he gets from the system. For him it is important to know, how reliable this planning is. The next steps will be the evaluation of feedback data depending on the source, which provides the information. Information of RFID (Radio Frequency Identification) devices is for example more reliable than manual feedback. With the evaluation of the received data the reliability of the implemented simulation model and thus the reliability of the planning can be evaluated.

\subsection{Different Scenarios of Planning}

Production processes are chaotic [18], which leads to a divergence that cannot be regarded completely in the planning. A small change of the initial value (e.g. the sequencing strategy of a single machine) can result over a longer period in large deviations. Therefore the prediction accuracy will decrease for longer forecast periods. For an accurate prediction, there are two conditions:

- A realistic description of the initial conditions, which is improved by the use of new sensor technologies and

- an exact model for the planning runs, which is more realistic due to cyber-physical systems (see 4.2). 
Since both conditions even in cyber-physical systems are never completely fulfilled in practice, prediction uncertainty arises inevitably. The next step is the determination of the most probable situation on the shop floor in the future. To determine the most probable situation on the shop floor a simulation model can be used whose initial conditions differ slightly from each other. Thus, faulty feedback data are relativized and the chaotic behavior of the production processes is taken into account. The further the forecast period goes into the future, the higher the differences of the individual predictions. The resulting forecast corridor indicates the most probable state of the production in the near future.

\section{Summary}

The described approach of cyber-physical production management is based on the abilities of cyber-physical systems. They enable the possibility to get better information due to the use of new sensor technologies and to run a huge number of simulations at the same time due to an increasing computational power. Therefore, deviations between today's detailed scheduling systems and the real situation on the shop floor, being described more detailed than before, can be determined. With this information better simulation models for planning runs can be depicted. By comparing differently parameterized simulation models with the real situation on the shop floor the best fitting model can be determined. With this model an optimized planning can be executed, which supports the practical planer efficiently. The next steps will be the evaluation of the performed planning by determining the quality of the input data and the probability of its reliability. Thus, the practical planner is provided and the increasing complexity becomes controllable. Finally, the efficiency of the production will be increased.

Acknowledgments. The presented approach of cyber-physical production management was developed within the research project "ProSense", which is funded by the German Federal Ministry of Education and Research (BMBF) within the Framework Concept "Research for Tomorrow's Production" (funding number 02PJ2490) and managed by the Project Management Agency Karlsruhe (PTKA).

\section{References}

1. Nyhuis, P., Münzberg, B., Kennemann, M.: Configuration and regulation of PPC. Production Engineering 3(3), 287-294 (2009)

2. Zaeh, M.: A holistic framework for enhancing the changeability of production systems. In: 1st Conference on Changeable, Agile and Virtual Production, CARV 2005, Munich, Germany (2005)

3. Lödding, H.: Handbook of Manufacturing Control, p. 2. Springer, Berlin (2012)

4. Reinhart, G., Gyger, T.: Identification of implicit strategies in production control. In: Proceedings of the Industrial Engineering and Engineering Management, IEEM, Singapore, pp. 302-306 (2008) 
5. Wiendahl, H.-P., ElMaraghy, H., Nyhuis, P., Zäh, M., Wiendahl, H.-H., Duffie, N., Brieke, M.: Changeable Manufacturing - Classification, Design and Operation. Annals of the CIRP 56(2), 783-809 (2007)

6. Friedli, T., Schuh, G., Kurr, M.: Kooperationsmanagement: Systematische Vorbereitung Gezielter Auf- und Ausbau - Entscheidende Erfolgsfaktoren, p. 34. Carl Hanser Verlag, München (2005)

7. Schuh, G., Gottschalk, S., Höhne, T.: High Resolution Production Management. CIRP Annals - Manufacturing Technology 56(1), 439-442 (2007)

8. Schuh, G., Franzkoch, B., Potente, T., Fuchs, S.: Simulation based configuration of value stream oriented production control. Production and Operation Management Society, POMS Vancouver (2010)

9. Zaeh, M., Reinhart, G., Ostgathe, M., Geiger, F., Lau, C.: A holistic approach for the cognitive control of production systems. Advanced Engineering Informatics 24(3), 300-307 (2010)

10. Gassmann, O., Kobe, C.: Management von Innovation und Risiko, p. 18. Springer, Berlin (2006)

11. Tempelmeier, H.: Supply chain planning with advanced planning systems. In: Proceedings of the 3rd Aegean International Conference on Design and Analysis of Manufacturing Systems, pp. 19-22 (May 2001)

12. Dimitrov, T., Baumann, M., Schenk, M.: Master data extraction and adaptation based on collected production data in manufacturing execution systems. In: 7th CIRP International Conference on Intelligent Computation in Manufacturing Engineering, CIRP ICME 2010, Capri, Italy, June 23-25 (2010)

13. Baheti, R., Gill, H.: Cyber-physical systems. The Impact of Control Technology, 161-166 (2011)

14. Broy, M. (ed.): Cyber-physical systems: Innovation durch softwareintensive eingebettete Systeme. Springer, Berlin (2010)

15. Vogel-Heuser, B., Bayrak, G., Frank, U.: Agenda CPS-Szenario smart factory. In: VogelHeusser, B. (ed.) Erhöhte Verfügbarkeit und transparente Produktion, pp. 6-21. Univ. Press, Kassel (2011)

16. Schuh, G., Stich, V.: Produktion am Standort Deutschland, pp. 16-17. Druckservice Zillekens, Aachen (2011)

17. Schuh, G., Potente, T., Hauptvogel, A.: A cyber-physical approach for production control based on second order cybernetics. In: CIRP January Meetings 2013, Paris, France, January 23-25 (2013)

18. Moon, F.: Dynamics and Chaos in Manufacturing Processes. Wiley Interscience (1997) 